livraisons

d'Histoire

de l'Architecture

\section{Livraisons de l'histoire de l'architecture}

41 | 2021

L'architecte en son agence

\title{
Krzysztof Pomian, Le musée, une histoire mondiale
}

Tome II : L'ancrage européen, 1789-1850, Paris, Gallimard, coll.

Bibliothèque illustrée des Histoires, 2021, 560 p.

\section{Baptiste Roelly}

\section{OpenEdition}

\section{Journals}

Édition électronique

URL : https://journals.openedition.org//ha/3358

DOI : $10.4000 /$ /ha.3358

ISSN : 1960-5994

Éditeur

Association Livraisons d'histoire de l'architecture - LHA

Référence électronique

Baptiste Roelly, «Krzysztof Pomian, Le musée, une histoire mondiale», Livraisons de l'histoire de l'architecture [En ligne], 41 | 2021, mis en ligne le 15 juin 2021, consulté le 18 juin 2021. URL : http:// journals.openedition.org//ha/3358; DOI : https://doi.org/10.4000//ha.3358

Ce document a été généré automatiquement le 18 juin 2021

Tous droits réservés à l'Association LHA 


\section{Krzysztof Pomian, Le musée, une histoire mondiale}

Tome II : L'ancrage européen, 1789-1850, Paris, Gallimard, coll. Bibliothèque illustrée des Histoires, 2021, 560 p.

\section{Baptiste Roelly}

1 Après un premier volume consacré à l'émergence des musées ( $\mathrm{XV}^{\mathrm{e}}$-XVIII ${ }^{\mathrm{e}}$ siècles) et avant un dernier consacré à leur globalisation (1851-aujourd'hui), Krzysztof Pomian livre le deuxième tome de son ambitieuse histoire mondiale des musées. De la Révolution française à la rivalité culturelle des États nationaux, le laps de temps ici traité voit la France prendre le pas sur l'Italie et devenir le pôle où les innovations muséales s'élaborent avant d'être diffusées à travers l'Europe. Si l'auteur inscrit toujours son sujet dans une perspective qui entrecroise histoire des mentalités, des systèmes de croyances, des modèles économiques et des structures politiques, sa méthodologie évolue quelque peu par rapport au premier tome pour se concentrer ici sur l'histoire institutionnelle de certains musées spécifiques. Son érudition, toujours critique, se nourrit alors de sources telles que les modalités d'accès aux collections ou les règles de conduite fixées aux visiteurs, pour en déduire les priorités que se donnent les institutions et comprendre l'expérience qu'en font les publics.

2 Son souci d'exhaustivité conduit l'auteur à retracer les débats autour de la question patrimoniale qui jalonnent la période révolutionnaire, dans la mesure où ils permettent l'émergence d'un nouveau modèle muséal. Tandis que la genèse du Louvre (successivement dénommé Muséum Français, Muséum national, Muséum central des arts et, plus tard, Musée Napoléon) est étudiée à la lumière des différents rapports de commissions ou débats parlementaires qui décident de la constitution de ses collections et de son installation, certaines personnalités font l'objet de focus particuliers pour avoir introduit des idées qui demeurent fondamentales aujourd'hui. Ainsi Pierre-Joseph Cambon fait-il figure de pionnier lorsqu'il comprend le musée comme un lieu neutre où d'anciennes reliques de la monarchie deviennent seulement des œuvres d'art. De même, l'insistance de Jean-Marie Roland de la Platière sur la nécessité qu'il y a de permettre à tout individu l'accès aux collections en fait le premier à aborder le musée 
comme une institution foncièrement démocratique. Une étude du partage de compétences et d'œuvres opéré entre le Louvre et Versailles démontre plus loin que le premier est compris comme un musée de chefs-d'œuvre universels, tandis que le second n'est dévolu qu'à l'école française. Deux orientations opposées en découlent, le Louvre accrochant ses œuvres selon des critères esthétiques et Versailles privilégiant un déroulé chronologique. "Né et mort de la guerre », le Musée Napoléon est abordé par le prisme des spoliations napoléoniennes qui modifient son contenu et pour son acception du musée comme instrument de propagande. En matière de politique muséale conduite par l'Empire, l'auteur rappelle à la suite d'Édouard Pommier que, contrairement à ce qui est souvent écrit, l'arrêté Chaptal de 1801 ne crée pas quinze musées de province : «dans neuf cas, il ne faisait que conférer une reconnaissance officielle aux musées qui existaient déjà. Dans d'autres, l'ouverture effective n'eut lieu que plusieurs années plus tard et, dans une ville, l'envoi reste sans effet. » Une autre vérité souvent occultée est rétablie au sujet du Musée des Monuments français, où l'intention d'Alexandre Lenoir n'est pas de se concentrer sur le Moyen Âge. Sa préférence va à la Renaissance, et la raison pour laquelle l'art médiéval trouve une place au couvent des Petits Augustins tient à ce que ce musée entend dérouler toute l'histoire nationale sans exception sous les yeux des visiteurs. Lenoir est en outre distingué ici comme le premier à comprendre que les débris du passé peuvent avoir une valeur historique, en ce que leur puissance évocatrice permet de revivre ce passé sur un mode imaginaire. Loin de se restreindre aux seuls musées d'arts ou d'antiques, Krzysztof Pomian consacre des développements analogues au Muséum national d'histoire naturelle, au Conservatoire national des arts et métiers, au Cabinet d'anatomie de l'École de santé de Paris et au musée de l'Artillerie. Tous résultent de la réorganisation révolutionnaire des institutions scientifiques françaises et connaissent un succès mesuré ici à l'aune des émules qu'ils suscitent à travers l'Europe. S'arrêtant à la chute de l'Empire en 1815 pour proposer un premier bilan, l'auteur relève que la croissance quantitative et la diversification thématique des musées s'accompagnent en ces années de la démocratisation de leur contenu. Ainsi le Conservatoire national des arts et métiers présente-t-il des machines, des outils et des objets de la vie quotidienne. Il semblerait en outre que ce soit dans le sillage de la Révolution et de la politique de pillage des pays étrangers que le musée devient l'objet d'une critique de principe et d'un débat intellectuel de fond. Quatremère de Quincy apparait sans surprise comme l'un de ses principaux instigateurs, mais l'auteur complexifie là aussi les choses en relativisant la muséophobie qui lui est habituellement prêtée. S'appuyant sur les Lettres à Miranda (1796), il démontre que Quatremère approuve la création des musées d'antiquités et reconnaît une vocation clairement didactique aux musées d'art. Ce qu'il rejette, c'est l'accaparement des arts et des sciences par une nation et le messianisme officiel français : « en prononçant la condamnation du musée en tant qu'établissement spécialisé, créé par une décision arbitraire du pouvoir, et rassemblant des œuvres séparées du contexte dans lequel s'est déroulé leur histoire ", Quatremère s'oppose moins aux musées en tant que tels qu'à la manière dont Dominique-Vivant Denon et Alexandre Lenoir les comprennent.

3 Après une première partie consacrée à la France révolutionnaire et impériale, l'auteur souligne le rôle joué par les musées dans la construction des identités nationales et propose un tour d'horizon de ceux qui ont vu le jour en Europe entre 1815 et 1850. L'Espagne présente à cet égard de nombreuses similitudes avec la France : le Prado résulte de la nationalisation des collections royales et les musées de province sont 
constitués à partir des œuvres d'art confisquées au clergé par l'État. L'auteur déduit des modalités d'accès au Prado que celui-ci entend d'abord permettre aux artistes d'étudier les meilleurs exemples de la peinture du passé, puis diffuser les gloires de la peinture espagnole à l'étranger, et enfin contribuer à l'éducation artistique de la population. Des considérations ayant trait à l'histoire du regard sont introduites ici, puisqu'une « salle réservée » hébergeant des nus qui ne pouvaient être vus sans autorisation spéciale existe au Prado entre 1827 et 1838. Elle est interprétée comme un signe de la lente transition qui permet au tableau de ne plus être regardé seulement comme une fenêtre ouverte sur le monde réel, mais apprécié pour ses qualités picturales propres et considéré comme un objet autonome. En Grande-Bretagne, l'auteur s'arrête sur la vogue antique notamment initiée par la Society of Dilettanti qui conduit le British Museum à se départir de ses collections d'histoire naturelle pour devenir un musée d'art antique. L'égyptomanie et le changement de statut que connaissent les antiquités égyptiennes après les travaux de Champollion sont retracés en détail : cessant d'être de simples monuments d'un art premier et des curiosités inintelligibles, elles entrent, grâce au déchiffrement des hiéroglyphes, dans le champ de la philologie et de l'histoire savante. Toujours plus ouverts à des œuvres étrangères aux canons de beauté grec, les musées abandonnent ainsi dans les années 1820 leur croyance en la validité d'une norme de beauté universellement valable. L'ouverture de la National Gallery en 1824 est également étudiée, de même que les multiples initiatives muséales privées (John Soane Museum, India Museum, Madame Tussaud,...) et le rôle des sociétés savantes qui soulignent tout au long du siècle combien l'histoire naturelle occupe une place prépondérante dans les loisirs des classes privilégiées. À propos de l'Allemagne, l'auteur insiste particulièrement sur le sentiment national qui nait avec les guerres de libération et imprègne la culture de manière croissante. Ce sentiment conduit de nombreux collectionneurs à doter leurs villes de musées, ainsi de Johann Friedrich Städel qui fonde avec le Städelsches Kunstinstitut de Francfort le premier musée d'art d'Allemagne en 1815. Soucieux de préserver le patrimoine religieux dispersé par la sécularisation, Ferdinand Franz Wallraf fait de même à Cologne et de nombreux individus ou Kunstvereine leur emboîtent le pas à Leipzig, Dresde, Nuremberg ou Mayence. Signe de la réévaluation des primitifs allemands, la collection réunie dans les mêmes années par les frères Boisserrée est achetée pour la Pinacothèque de Munich qui ouvre en 1836. Celle-ci innove en abandonnant le modèle parisien de la longue et unique galerie, lui préférant des salles en enfilade. Ses visiteurs remarquent dans leurs récits combien cet agencement met en valeur les tableaux dans un espace qui leur est exclusivement adapté, l'un d'eux louant Leo von Klenze pour avoir conçu à Munich le réceptacle de tableaux le plus approprié qui soit en Europe. La galerie de Berlin introduit elle aussi une nouveauté en adoptant le principe de la cohabitation des artistes dans le temps en matière d'accrochage des œuvres. Si certains visiteurs désapprouvent l'abandon du seul critère de l'origine géographique des artistes, cet éclatement de la notion traditionnelle d'école due à Gustav Friedrich Waagen n'en traduit pas moins une approche d'historien de l'art qui rompt avec les pratiques d'amateurs jusqu'alors privilégiées. Du côté des musées d'histoire, les innovations conduites par Christian Jürgen Thomson permettent à l'auteur d'affirmer que le Danemark fait figure de pionnier pour presque tout ce qui touche aux musées d'histoire. Un parallèle inédit est en outre établi entre le Walhalla, voulu par Louis Ier de Bavière, et le musée de l'Histoire de France du château de Versailles. Voulu dès 1830 par Louis-Philippe, ce dernier démontre combien la politique culturelle de la 
Restauration s'inscrit dans la continuité de celles conduites sous la Révolution et l'Empire. Il répond à un besoin de concevoir des musées où le passé de la nation devient visible, lequel besoin présidait déjà à la création du musée des Monuments français. La disparition du musée d'Alexandre Lenoir en 1816 laisse à cet égard un vide que comble la création du musée de Cluny en 1843. Parmi les autres musées créés sous la Restauration, l'auteur relève que le musée de la Céramique de Sèvres ouvert en 1824 est alors le premier musée des arts décoratifs de l'histoire et que la conception du musée de la Marine (1827) offre leurs prémices aux débats sur les musées d'ethnologie.

4 Les apports de cette nouvelle histoire des musées sont, en somme, nombreux et fondamentaux. Ils soulignent la capacité déjà largement reconnue de son auteur à aborder de concert des champs historiographiques et des méthodologies habituellement traitées à part. L'histoire du goût lui permet, par exemple, de poser un regard nouveau sur un pan de l'histoire institutionnelle, de la même manière que l'étude de la réception des musées nourrit celle de leur inscription idéologique. Krzysztof Pomian opère pour ce faire des allers-retours entre macro (guerres napoléoniennes, sécularisation, industrialisation...) et micro-histoire (principes déterminant l'accrochage des œuvres, récits de voyages et correspondances...), situant son objet d'étude à un carrefour disciplinaire où interviennent tant l'anthropologie et l'histoire de l'art que la sociologie et l'histoire économique. 